\title{
The Two-impurity Anderson Model Revisited: Competition between Kondo Effect and Reservoir-mediated Superexchange in Double Quantum Dots
}

\author{
Minchul Lee, ${ }^{1,2}$ Mahn-Soo Choi, ${ }^{1, \text { ॠ }}$ Rosa López, ${ }^{3}$ Ramón Aguado, ${ }^{4}$ Jan Martinek, ${ }^{5}$ and Rok Žitko ${ }^{6}$ \\ ${ }^{I}$ Department of Physics, Korea University, Seoul 136-713, Korea \\ ${ }^{2}$ Department of Applied Physics, Kyung Hee University, Yongin 449-701, Korea \\ ${ }^{3}$ Departament de Física, Universitat de les Illes Balears, E-07122 Palma de Mallorca, Spain \\ ${ }^{4}$ Teoría de la Materia Condensada, Instituto de Ciencia de Materiales de Madrid (CSIC) Cantoblanco, 28049 Madrid, Spain \\ ${ }^{5}$ Institute of Molecular Physics, Polish Academy of Sciences, Smoluchowskiego 17, 60-179 Poznań, Poland \\ ${ }^{6}$ J. Stefan Institute, Jamova 39, SI-1000 Ljubljana, Slovenia
}

(Dated: October 22, 2018)

\begin{abstract}
We study a series-coupled double quantum dot in the Kondo regime modeled by the two-impurity Anderson model and find a new conduction-band mediated superexchange interaction that competes with Kondo physics in the strong Coulomb interaction limit. Our numerical renormalization group results, complemented with the higher-order Rayleigh-Schrödinger perturbation theory, show that the novel exchange mechanism leads to clear experimental consequences that can be checked in transport measurements through double quantum dots.
\end{abstract}

PACS numbers: 73.21.La; 73.23.-b; 73.22.-f

Quantum dots (QDs) [1] behave as quantum impurities, thus they exhibit the Kondo effect, the most spectacular manifestation of which is the transition from near-zero conductance due to Coulomb blockade to perfect transmission [2, 3] as the temperature is lowered well below the Kondo temperature, $T_{K}$. More complex setups allow tailored realizations of strongly correlated electron systems in new situations. Double quantum dots (DQDs) [4, 5, 6, 7], for instance, are a minimal system for studying a lattice of magnetic impurities in a tunable environment. In the two-impurity Kondo model, the competition between the Kondo effect and the antiferromagnetic (AF) interaction between impurities leads to a second-order quantum phase transition (QPT) [8]. It is also known that the QPT is replaced by a crossover if electrons can tunnel between the impurities [9]. In series-coupled DQDs, the most important contribution to the total AF exchange coupling is the superexchange coupling $J_{U} \approx 4 t^{2} / U$, where $t$ is the inter-dot hopping and $U$ the on-site interaction. This superexchange vanishes $\left(J_{U} \rightarrow 0\right)$ as $U \rightarrow \infty$, which leads to the common belief [4, 7] that the Kondo physics always prevail in the large- $U$ limit. In this Letter, we present numerical renormalization group (NRG) calculations which confute the above claim and unambiguously demonstrate the presence of a new type of spin-spin interaction which competes with Kondo physics in the large- $U$ limit. Our main findings are illustrated in Fig. 4 4 where the peak position of the linear conductance through a DQD versus $t$ exhibits a striking and unexpected dependence on $\Gamma$, the QD level broadening due to the coupling to the reservoir. Interestingly, the novel spin-spin interaction reported here leads to observable experimental consequences like zero conductance $G \approx 0$ in regions where unitary transport $G \approx 2 e^{2} / h$ is expected and vice versa. Intuitively, we can understand this spin-spin interaction in terms of virtual tunneling events involving conduction-band electrons; see Fig. 11. The strength of this spin-spin interaction, which we call conduction-band-mediated superexchange, $J_{I}$, can be estimated in the large- $U$ limit using Rayleigh-Schrödinger per-

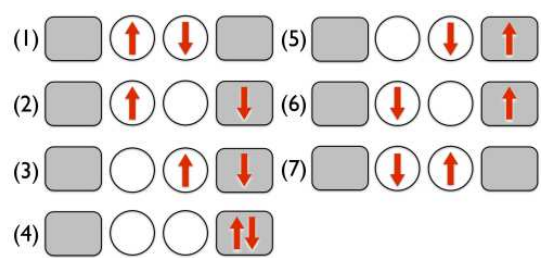

FIG. 1: The sequence of six virtual processes of the most significant contribution to the conduction-band-mediated exchange $J_{I}$ in the large- $U$ limit. Here the circles (rectangles) denote dots (leads).

turbation theory (RSPT) as

$$
J_{I}=\frac{4 t^{2} \Gamma^{2}}{\pi^{2}} \int_{E_{F}}^{D} \frac{d \epsilon_{1} d \epsilon_{2}}{\left(\epsilon_{1}-\epsilon\right)^{2}\left(\epsilon_{2}-\epsilon\right)^{2}\left(\epsilon_{1}+\epsilon_{2}-2 \epsilon\right)}
$$

for $t \ll|\epsilon|$ where $\epsilon<0$ is the single-particle energy level of QD. Here $E_{F}=0$ and $D$ are the Fermi energy and the halfwidth of the conduction band, respectively. For a wide band $(|\epsilon| \ll D)$, it simplifies to $J_{I} \approx c t^{2} \Gamma^{2} /|\epsilon|^{3}$ with a constant $c=8(1-\ln 2) / 3 \pi^{2} \approx 0.083$. Interestingly, a single lead is sufficient to induce a finite exchange $J_{I} \neq 0$, as seen in Fig.1. Remarkably, this high-order tunneling process is able to affect the transport properties of DQDs. In what follows, we present in detail our NRG study and discuss thoroughly the conditions under which $J_{I}$ can compete with Kondo physics in regions where $J_{U}$ vanishes.

Model and methods. - We model the DQD by the twoimpurity Anderson model:

$$
\begin{aligned}
& \mathcal{H}=\sum_{\ell \mathbf{k} \mu} \epsilon_{\mathbf{k}} c_{\ell \mathbf{k} \mu}^{\dagger} c_{\ell \mathbf{k} \mu}+\sum_{\ell}\left(\epsilon n_{\ell}+U n_{\ell \uparrow} n_{\ell \downarrow}\right) \\
& -t \sum_{\mu}\left(d_{1 \mu}^{\dagger} d_{2 \mu}+\text { h.c. }\right)+\sum_{\ell \mathbf{k} \mu} V\left[c_{\ell \mathbf{k} \mu}^{\dagger} d_{\ell \mu}+\text { h.c. }\right]
\end{aligned}
$$

Here $c_{\ell \mathbf{k} \mu}$ destroys a spin- $\mu$ electron with energy $\epsilon_{\mathbf{k}}$ in the lead $\ell=1,2$, and $d_{\ell \mu}$ an electron in the $\operatorname{dot} \ell=1,2 ; n_{\ell} \equiv$ 
$\sum_{\mu} d_{\ell \mu}^{\dagger} d_{\ell \mu}$ is the occupation of the $\operatorname{dot} \ell$. For simplicity, we assume reflection symmetry with respect to the interchange $1 \leftrightarrow 2$ of leads and dots. The single-particle energy $\epsilon$ on each dot is tunable by gate voltages. The hybridization between the dot and lead is characterized by $\Gamma=\pi \rho V^{2}$, with a flat-band density of states $\rho=1 / 2 D$. Throughout this work, we fix $\epsilon=-0.1 D$ and focus on the Kondo regime with localized level, $\Gamma \ll|\epsilon|$ and large charging energy $U \geq 2|\epsilon|$.

We solve the Hamiltonian with the standard NRG procedure [10, 11, 12]. At low temperatures, a Fermi-liquid system is described by an effective Hamiltonian that takes a form similar to the original Hamiltonian but with renormalized parameters [13]. Previous works have applied this theoretical approach to deal with single dots [13]. Here we extend it to treat the two-impurity problem of a DQD. It is then technically convenient to change to the parity basis (even and odd): $c_{e(o) \mathbf{k} \mu}=\left(c_{1 \mathbf{k} \mu} \pm c_{2 \mathbf{k} \mu}\right) / \sqrt{2}$ and $d_{e(o) \mu}=\left(d_{1 \mu} \pm d_{2 \mu}\right) / \sqrt{2}$. In this basis, the Hamiltonian in Eq. (2) reads as

$$
\begin{array}{r}
\mathcal{H}=\sum_{s=e, o}\left[\sum_{\mathbf{k} \mu} \epsilon_{\mathbf{k}} c_{s \mathbf{k} \mu}^{\dagger} c_{s \mathbf{k} \mu}+\epsilon_{s} n_{s}+\frac{U}{2} n_{s \uparrow} n_{s \downarrow}\right] \\
+\frac{U}{4} n_{e} n_{o}-U \mathbf{S}_{e} \cdot \mathbf{S}_{o}-\frac{U}{2}\left[d_{e \uparrow}^{\dagger} d_{e \downarrow}^{\dagger} d_{o \downarrow} d_{o \uparrow}+h . c .\right] \\
+\sum_{s \mathbf{k} \mu} V\left[c_{s \mathbf{k} \mu}^{\dagger} d_{s \mu}+h . c .\right]
\end{array}
$$

Here the local spin operator is defined by $\mathbf{S}_{s}=$ $\frac{1}{2} \sum_{\mu \mu^{\prime}} \sigma_{\mu \mu^{\prime}} d_{s \mu}^{\dagger} d_{s \mu^{\prime}}$ with $\sigma$ being Pauli matrices, and the even (odd) level is given by $\epsilon_{e(o)}=\epsilon \mp t$.

The renormalized parameters at the Fermi-liquid fixed point (which we denote by appending an asterisk to bare parameter symbols) are extracted from the NRG flow diagrams. Additional terms, missing in the bare Hamiltonian, which are allowed by the symmetry are generated in the renormalization process. In the absence of the particle-hole (p-h) symmetry, for example, coefficients $U_{s}$ of the term $n_{s \uparrow} n_{s \downarrow}$, hybridization $\Gamma_{s}$, and energies $\epsilon_{s}$ will renormalize differently in each parity channel. In the p-h symmetric case we were able to determine all interaction terms in the local basis, in particular, the exchange coupling $J_{12}^{*}$ between dot spins $\mathbf{S}_{1}$ and $\mathbf{S}_{2}$. Finally, the linear conductance is determined by even and odd quasi-particle scattering phase shifts as $G=$ $\left(2 e^{2} / h\right) \sin ^{2}\left(\delta_{e}-\delta_{o}\right)$ [4]. In the following we consider two cases: (i) the model with the p-h symmetry and intermediate$U$, where the physics is dominated by $J_{U}$ and (ii) the large$U$ case where $J_{U}$ is suppressed. Analysis of two cases reveals the emergence of a new type of anti-ferromagnetic coupling $J_{I}$ generated by high-order virtual tunneling events (see Fig. (1) among the electrons localized on dots and the itinerant carriers in the reservoirs.

Particle-hole symmetric, intermediate-U regime.- Let us review briefly the main features for the p-h symmetric case in Fig. 2. The Friedel-Langreth sum rule imposes the relation $\delta_{e}+\delta_{o}=\pi$ for all $t$ and then the average occupation per spin channel is one. The physics is governed by the com-

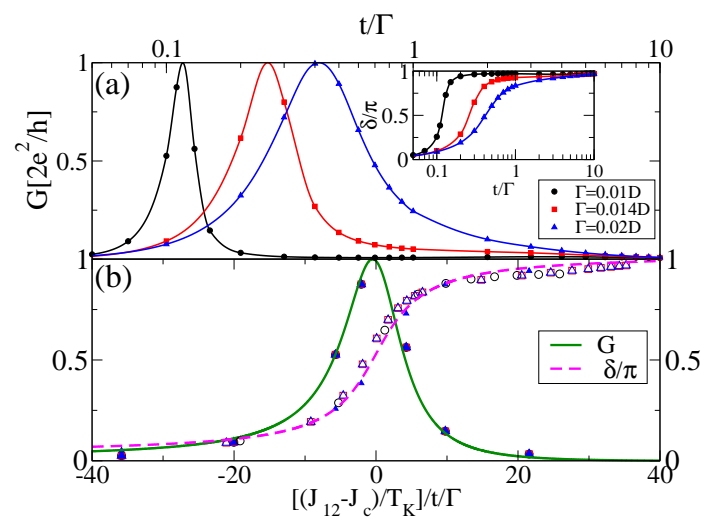

FIG. 2: (a) Zero-temperature linear conductance and phase shift difference $\delta=\delta_{e}-\delta_{o}$ in the symmetric case (inset) as functions of $t / \Gamma$, $U=2|\epsilon|$. Lines are guidance for eyes. (b) Linear conductance $G$ (filled symbols) and phase shift as functions of the scaled exchange coupling, $\left(J_{12}-J_{c}\right) \Gamma / T_{K} t$. Results for the phase shift differences and for conductance collapse well onto universal curves, the scaling functions fitted to the data.

petition between the Kondo and the AF energy scales. For small $t, J_{12} \ll T_{K}$ and each dot spin forms a Kondo singlet state with conduction electrons in the neighboring lead, while the weak inter-dot coupling yields small conductance. Furthermore, the spin-spin correlation $\left\langle\mathbf{S}_{1} \cdot \mathbf{S}_{2}\right\rangle$ vanishes; see Fig. 3a). As $t \rightarrow \infty, J_{12} \gg T_{K}$, and the dot spins are locked into a local singlet state, thus inhibiting the Kondo effect, and consequently the conductance is small in this limit as well. As $t$ increases from 0 to $\infty$, the conductance exhibits one pronounced peak as shown in Fig. 22 a) where the peak position is determined by the condition $J_{c}\left(t=t_{c}\right)=J_{12} \sim T_{K}$. At this point the spin correlation takes the value $\left\langle\mathbf{S}_{1} \cdot \mathbf{S}_{2}\right\rangle=-1 / 4$ due to the singlet-triplet degeneracy [8]. The crossover occurs from the Kondo to AF regime [4, 9] where $J_{12} \sim T_{K}$, and the parameters $t^{*}$ and $\Gamma^{*}$ become equal at $J_{12}=J_{c}$. This crossover is known to be well described by a scaling function [4]: $\delta / \pi=\phi\left[\left(J_{12}-J_{c}\right) \Gamma / T_{K} t\right]$ with $\phi(-\infty)=0$ and $\phi(\infty)=1$ which is in good agreement with our numerical results; see Fig. 22 (b).

Large- $U$ regime.- We found that the large- $U$ case is governed by spin-spin correlations mediated by the conductionband electrons. The linear conductance features a plateau starting at $t \sim|\epsilon|[14]$ and a peak at lower $t$; see Fig. 4. Importantly, the peak reaches the unitary limit and shifts toward larger $t / \Gamma$ with increasing $\Gamma$ as in the symmetric case. It must be emphasized that this behavior of the conduction peak is wholly unexpected and disagrees with previous analyzes for the $U \rightarrow \infty$ limit. We therefore continue with a more detailed study of this feature. In the large- $U$ limit, the usual superexchange $J_{U} \propto 1 / U$ vanishes. For this reason, the conductance peak has been traditionally ascribed to the formation of even and odd Kondo states occurring at $\Gamma^{*}=t^{*}$. In addition, some theories [4, 15] predicted that $\Gamma$ and $t$ are renormalized in the same manner, thus the transition from individual Kondo states to bonding and anti-bonding states was 


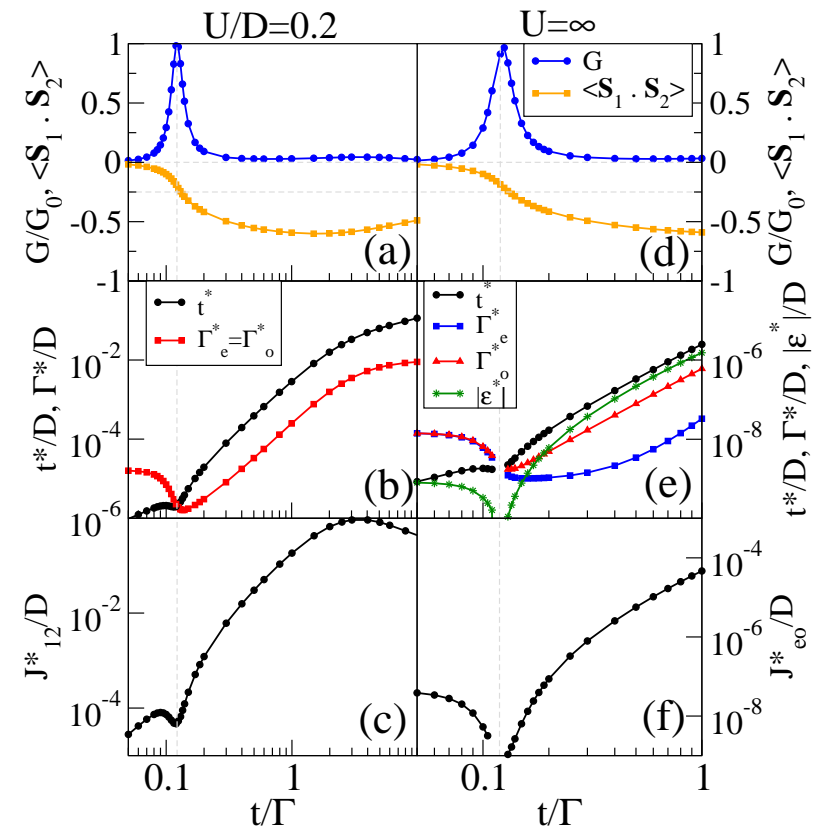

FIG. 3: Zero-temperature properties of the p-h symmetric model (left panels) and the p-h asymmetric $U=\infty$ model (right panels) for $\Gamma=$ $0.01 D$. (a,d) Linear conductance and spin-spin correlation $\left\langle\mathbf{S}_{1} \cdot \mathbf{S}_{2}\right\rangle$. (b,e) Renormalized parameters, $t^{*}, \Gamma_{e}^{*}, \Gamma_{o}^{*},\left|\epsilon^{*}\right|$. In the symmetric model, $\Gamma_{e}^{*}=\Gamma_{o}^{*}$ and $\epsilon^{*}=0$. (c,f) Renormalized superexchange coupling in the local basis (c) and in the parity basis (f).

expected to happen at $\Gamma=t$. However, the results in Fig. 4 are in clear disagreement with this picture: the conductance peak is not located at $\Gamma=t$, but rather at $t<\Gamma$ and it is shifted toward smaller $t / \Gamma$ with decreasing $\Gamma$. This suggests the presence of some processes which are responsible for the different renormalization of $\Gamma$ and $t$. We indeed find an exchange coupling between the dot spins, which is manifested in the unexpectedly strong anti-ferromagnetic correlation $\left\langle\mathbf{S}_{1} \cdot \mathbf{S}_{2}\right\rangle<0$ in Fig. 3 d). At the conductance peaks, this correlation takes the value of $-1 / 4$. The origin of the exchange coupling at $U \rightarrow \infty$ is illustrated in Fig. 1 in which the dot spins get to be locked into a singlet state by means of virtual tunneling processes where conduction-band electrons participate.

The strength of this conduction-band-mediated superexchange coupling $J_{I}$ in the large- $U$ limit is estimated using the RSPT; see Eq. (1). The RSPT (or projection method) should be enough to extract the almost true value of $J_{I}$ since $J_{I}$ is due to the charge fluctuations, as $J_{U}$ is; see Fig. 11. Further scaling would affect only the spin fluctuation part related to the Kondo effect and not to the local spin interactions. Hence, $J_{I}$ computed from the RSPT should be, at least in order of magnitude, very close to the one that competes with the Kondo scale. The superexchange coupling $J_{I}$ is generated at the fourth order of $V$ in the RSPT scheme and in the limit $t \ll|\epsilon|, U$ it is of the second order in $t$; see Eq. (1). It is remarkable that such higher-order processes lead to sufficiently strong spin correlations to qualitatively affect the transport properties of the $D Q D$. The maximal conductance at the crossover region oc-

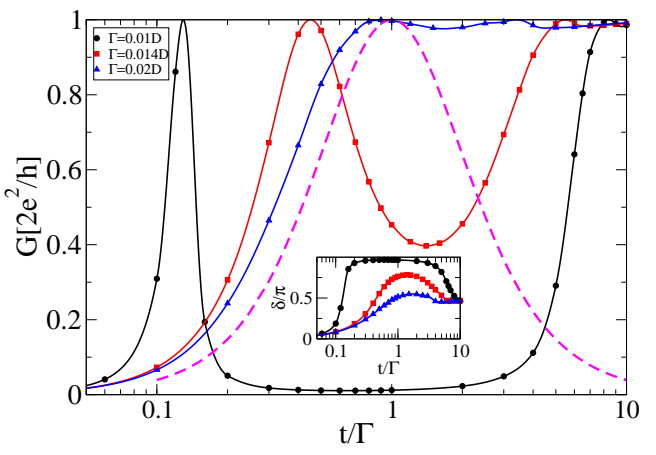

FIG. 4: Zero-temperature linear conductance and phase-shift difference $\delta=\delta_{e}-\delta_{o}$ (inset) in the large- $U$ case $(U=\infty)$. Lines are guidance for eyes. The dashed line is obtained from the SBMFT calculation [4].

curs because $J_{I}$ drives the system from the Kondo phase to the anti-ferromagnetic phase at $J_{I} \approx T_{K}$, in full analogy with the symmetric case. This is underpinned by the observation that the renormalized $\epsilon^{*}$ goes through zero, even and odd hybridizations become equal, $\Gamma_{e}^{*}=\Gamma_{o}^{*}=\Gamma^{*}$, and $\Gamma^{*}=t^{*}$; see Fig. 3(e). At this particular point, the p-h symmetry is, surprisingly, restored at low energies. We also find that the magnetic interaction $J_{e o}^{*}$ (it is technically difficult to extract $J_{12}^{*}$ unlike in the p-h symmetric case) again exhibits a local minimum at the cross over [see Fig. 3(f)]. The fact that the conductance peak is shifted away from $\Gamma=t$ can be regarded as the experimental proof of the existence of a new type of spin-spin correlations not accounted so far. Other theoretical techniques like slave-boson decomposition [4, 15] do not describe properly these magnetic correlations between the dot spins that arise naturally as the interplay between the tunneling events and the Coulomb repulsion. In these approaches, spin exchange interactions are usually included ad hoc [12]. Our results suggest that both $J_{U}$ and $J_{I}$ have to be included in slave-boson schemes in order to achieve a good agreement with NRG calculations.

Crossover- Now we address the intermediate regime with medium $U$ where both anti-ferromagnetic couplings are finite. After a lengthy calculation it is possible to obtain an analytical expression for $J_{I}$ as a function of $U[16]$. The expression is too cumbersome to appear here and we just plot the final results. The inset in Fig. 5] shows the relative portion of $J_{I}$ in the total superexchange versus $U$. For sufficiently large $U, J_{I}$ surely dominates over $J_{U}$ that eventually vanishes as $U \rightarrow \infty$. For intermediate values of $U \lesssim 10 D$, $J_{I} \ll J_{U}$ the physics is governed by the superexchange interaction $J_{U}$, while it is $J_{I}$ that suppresses the Kondo correlations for larger $U$. Since $J_{I}$ is proportional to $\Gamma^{2}$, the value of $U$ where $J_{I}$ becomes dominant over $J_{U}$ can be lower with increasing $\Gamma$. The competition between $J_{I}$ and $J_{U}$ are also reflected in the main plot of Fig. 5] where the linear conductance peak position versus $U$ is plotted. While for intermediate $U$ the peak position is well identified by the condition $J_{U} \sim T_{K}$ (dotted lines), which is in agreement with previ- 


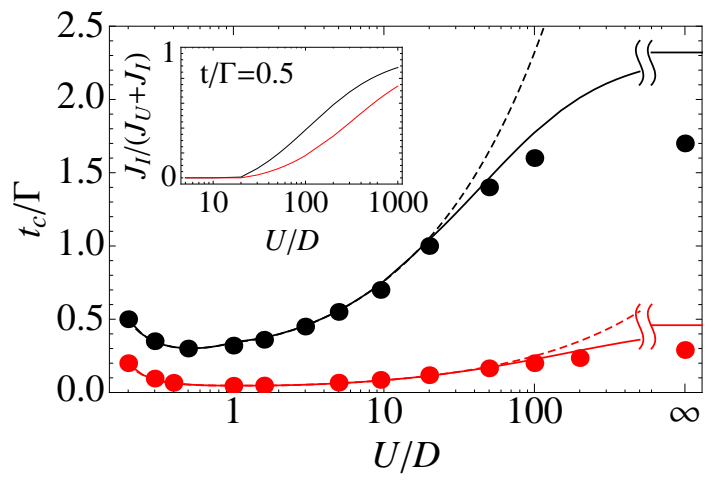

FIG. 5: The conductance-peak position $t_{c} / \Gamma$ as a function of the Coulomb energy $U$ for different values of $\Gamma: \Gamma / D=0.012$ (red) and 0.02 (black). The dotted and solid lines are the peak positions estimated from the condition $J_{U}=2.2 T_{K}$ and $J_{U}+J_{I}=2.2 T_{K}$, respectively. Inset: the relative strength of $J_{I}$ with respect to the total superexchange coupling.

ous theories, the deviation becomes significant for large $U$, and in the $U \rightarrow \infty$ limit the peak position is given by the condition $J_{I} \sim T_{K}$. Interestingly, a good fitting for arbitrary value of $U$ is obtained by determining the peak position by imposing the condition $J=J_{I}+J_{U}=2.2 T_{K}$, with $T_{K}=\sqrt{\Gamma \min (U, D) / 2} \exp [2 \pi \epsilon(1+\epsilon / U) / \Gamma]$. Thus, the conduction-band-mediated superexchange explains in a very suitable manner (both qualitatively and quantitatively) the saturation of the conductance peak as $U$ is increased. Our estimation of the peak position from the RSPT is slightly larger than one obtained from the NRG calculations. It is due to our neglect of further scaling of $J_{I}$ with integration of the conduction band and the renormalization of $T_{K}$ due to the inter-dot coupling. Both renormalizations should be quite marginal and may lead to slightly larger $J_{I}$ and smaller $T_{K}$, with which the condition $J=2.2 T_{K}$ gives rise to smaller conductance peak position.

Discussion. - The RSPT estimation predicts that $J_{I}$ becomes comparable to $J_{U}$ at $U \approx U_{c}=4|\epsilon|^{3} / c \Gamma^{2}$. The ratio $U_{c} /|\epsilon|$ is then of the order of a few hundreds in order to retain the Kondo effect $(|\epsilon| / \Gamma \ll 1)$. The condition can be satisifed through the electric control of the single-particle level in tunable samples, or by making use of ultrasmall samples with strong Coulomb interaction. One of the latter canditates is a junction made of molecular dimers where $U$ is about a few $\mathrm{eV}$. While the interdot coupling of dimers can hardly be tuned, the gate voltage control of $\epsilon$ and $\Gamma$ can nevertheless fulfill the crossover condition $J_{I} \sim T_{K}$, allowing to examine the role of the superexchange on the transport.

Conclusion.- We have reported on a new conductionband mediated superexchange interaction, $J_{I}$, that competes with Kondo physics in the large- $U$ limit of the two-impurity problem. The novel exchange mechanism brings more unity to this problem in terms of magnetic correlations, and the transport through the DQD can be analyzed in terms of the competition between the Kondo correlation and the anti- ferromagnetic interaction for all values of $U$. Transport experiments can confirm the presence of high-order superexchange coupling by examining the dependence of the peak position on the inter-dot hopping amplitude.

R.A. acknowledges financial support from grant MAT200603741 (MEC-Spain) and CCG08-CSIC/MAT-3775. M.-S.C. was supported by the grant R11-2000-071, the BK21, and the KIAS; and J.M. by the Polish grant for science in years 20062009 as a research project; and R.L by the grant FIS200800781 (MEC-Spain).

* Electronic address: choims@korea.ac.kr

[1] For a review see, L. P. Kouwenhoven, et al., in Mesoscopic Electron Transport, edited by L. L. Sohn, L. P. Kouwenhoven, and G. Schön (Kluwer, Amsterdam, The Netherlands, 1997).

[2] L. I. Glazman and M. É. Ra ̌ikh, Pis'ma Zh. Eksp. Teor. Fiz. 47, 378 (1988) [JETP Lett. 47, 452 (1988)]; T. K. Ng and P. A. Lee, Phys. Rev. Lett.61, 1768 (1988).

[3] D. Goldhaber-Gordon, et al., Nature 391, 156 (1998); S. M. Cronenwett, T. H. Oosterkamp, and L. P. Kouwenhoven, Science 281, 540 (1998); J. Schmid, et al., Physica B 256258, 182 (1998); W. G. van der Wiel, et al., Science 289, 2105 (2000).

[4] A. Georges and Y. Meir, Phys. Rev. Lett. 82, 3508 (1999); T. Aono and M. Eto, Phys. Rev. B 63, 125327 (2001); R. Aguado, and D. C. Langreth, Phys. Rev. Lett. 85, 1946 (2000); R. Lopez, R. Aguado, and G. Platero, Phys. Rev. Lett. 89, 136802 (2002).

[5] H. Jeong, A. M. Chang, M. R. Melloch, Science 293, 2221 (2001); J. C. Chen, A. M. Chang, and M. R. Melloch, Phys. Rev. Lett. 92, 176801 (2004).

[6] N. J. Craig, et al., Science 304, 565 (2004).

[7] R. Lopez, P. Simon, and Y. Oreg, Phys. Rev. Lett. 94, 086602 (2005); R. M. Konik, Phys. Rev. Lett. 99, 076602 (2007).

[8] B. A. Jones, C. M. Varma, and J. W. Wilkins, Phys. Rev. Lett. 61, 125 (1988); B. A. Jones and C. M. Varma, Phys. Rev. B 40, 324 (1989).

[9] B. A. Jones, G. Kotliar, and A. J. Millis, Phys. Rev. B 39, 3415 (1989); G. Zarand, C.-H. Chung, P. Simon, and M. Vojta, Phys. Rev. Lett. 97, 166802 (2006).

[10] K. G. Wilson, Rev. Mod. Phys. 47, 773 (1975); H. R. Krishnamurthy, J. W. Wilkins, and K. G. Wilson, Phys. Rev. B 21, 1003 (1980).

[11] O. Sakai and Y. Shimizu, J. Phys. Soc. Jpn. 61, 2333 (1992).

[12] W. Izumida, O. Sakai, and Y. Shimizu, J. Phys. Soc. Jap. 66, 717 (1997).

[13] A. C. Hewson, A. Oguri, and D. Meyer, Eur. Phys. J. B 40, 177 (2004); A. Oguri, Y. Nisikawa, and A. C. Hewson, J. Phys. Soc. Jpn. 74, 2554 (2005); A. Oguri and A. C. Hewson, J. Phys. Soc. Jpn. 74, 988 (2005); A. C. Hewson, J. Phys.: Condens. Matter, 18, 1815 (2006); A. C. Hewson, J. Bauer, and W. Koller, Phys. Rev. B 73, 045117 (2006).

[14] The conductance plateau for large $t$ in Fig. 4 is due to oneelectron $S=1 / 2$ Kondo effect. The one-electron even-orbital state is lowered below the two-electron states when $t \gtrsim|\epsilon|$. Hence the conductance plateau is attributed to the formation of a single-impurity Kondo state in the even channel, and the conductance therefore reaches the unitary limit at zero temperature, independent of the value of $t / \Gamma$. 
[15] See, e.g., N. E. Bickers, Rev. Mod. Phys. 59, 845 (1987).

[16] Explicit expression for $J_{I}(U)$ will be given elsewhere. 